\title{
Miniaturised Ceramic Packages for Piezoresistive Pressure Sensors
}

\author{
Dr. Christian Wohlgemuth, Dr. Anton Leidl, Dr. Gregor Feiertag, Dipl.-Ing. Wolfgang Pahl \\ EPCOS AG \\ St.-Martin-Strasse 53 \\ 81669 München, GERMANY
}

\begin{abstract}
The precision of piezoresistive pressure transmitters is often limited by packaging effects. Especially the mounting of the sensor die plays a decisive role. While non-linearity and temperature dependence of the MEMS sensor itself can be compensated by signal conditioners, this is not possible for hysteresis and drift effects caused by inadequate die mounting. Coatings on the sensor die also emerge measurement errors.

In this paper the effects of mounting on the sensor die are described and two different of pressure sensors in ceramic packages are presented. One small sized differential pressure sensor with high accuracy and one extremely miniaturized barometric absolute pressure sensor especially designed for high volume processability.
\end{abstract}

\section{Introduction}

In the last 50 years the piezoresistive pressure sensor elements were developed continuously and are now the most frequently used pressure sensors in various applications. The design of this MEMS and the fabrication processes have reached a high level combining excellent measurement properties with low prices [1]. Because of the mono crystalline structure piezoresistive silicon sensor elements show a small hysteresis error and good long term stability. These properties are restricted by the media leading the pressure. Especially the topside of the sensor element with metallized conductive lines, doped resistor areas and wire bonds is sensitive for corrosion effects. For less aggressive media a gel coating of silicone or fluorine silicone can protect the sensor against environmental conditions. Fig. 1 gives an overview of typical housings of piezoresistive pressure sensors [2].
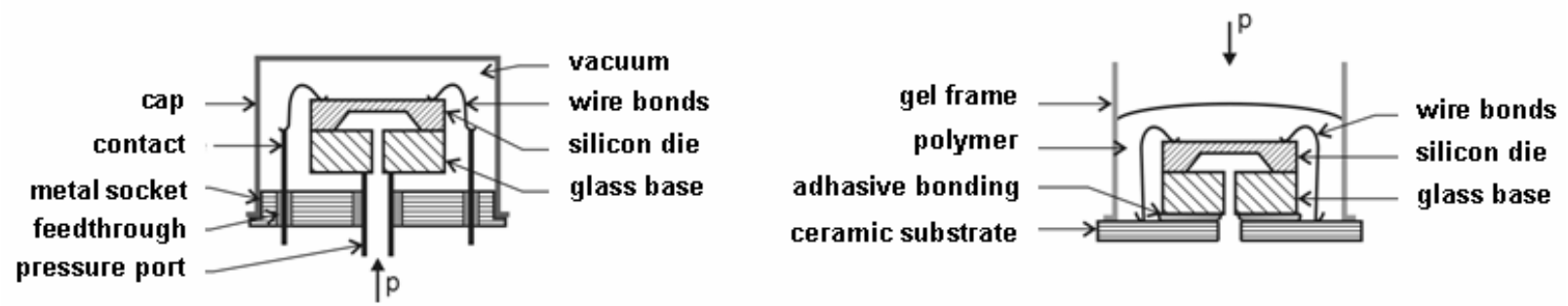

Fig. 1 Overview of pressure sensorss with piezoresistive sensor element

The quality of the output signal of pressure transmitters was tremendous improved with the development of integrated programmable signal conditioners compensating the temperature dependence and the nonlinearity of the transfer behavior. Only the effects of hysteresis and drift cannot be compensated. Sensor element and signal amplifying electronic are combined in one housing for the functional unit of the pressure transmitter, comprising the pressure ports and the electrical connections. Table 1 gives a survey of the origins of measuring errors of pressure transmitters with piezoresistive sensor elements.

Table 1 Survey of origins of measuring error

\begin{tabular}{|l|l|l|l|l|}
\hline Origin of error & correctable error & $\begin{array}{l}\text { quantity } \\
\text { of error }\end{array}$ & not correctable error & $\begin{array}{l}\text { quantity } \\
\text { of error }\end{array}$ \\
\hline piezoresistive sensor element & $\begin{array}{l}\text { offset } \\
\text { sensitivity } \\
\text { linearity }\end{array}$ & $\begin{array}{l}\text { high } \\
\text { medium } \\
\text { low }\end{array}$ & $\begin{array}{l}\text { hysteresis error } \\
\text { drift }\end{array}$ & $\begin{array}{l}\text { low } \\
\text { low }\end{array}$ \\
\hline mounting of the sensor element & offset & high & hysteresis & high \\
\hline
\end{tabular}




\begin{tabular}{|l|l|l|l|l|}
\hline & & & drift & high \\
\hline $\begin{array}{l}\text { encapsulation of the sensor } \\
\text { element }\end{array}$ & offset & low & $\begin{array}{l}\text { hysteresis } \\
\text { drift }\end{array}$ & $\begin{array}{l}\text { medium } \\
\text { medium }\end{array}$ \\
\hline signal conditioner & $\begin{array}{l}\text { internal errors } \\
\text { AD-sampling }\end{array}$ & $\begin{array}{l}\text { low } \\
\text { medium }\end{array}$ & $\begin{array}{l}\text { quantisation noise } \\
\text { sapling errors }\end{array}$ & $\begin{array}{l}\text { low } \\
\text { low }\end{array}$ \\
\hline
\end{tabular}

The main origin of not correctable errors is the mounting of the sensor element. The design of this feature has to be improved carefully. The protection against environmental stress by a gel coating or by media separation has a smaller influence.

\section{Mounting of piezoresistive sensor elements}

Mechanical tensions implied at the mounting patch, go through the hole sensor element and cause variations of the piezoresistive Wheatstone bridge. A not pressure dependent signal, an error signal, is generated [4].

The measurement errors of mounting are caused by the thermal mismatch between the sensor element, the substrate and the adhesive. Table 2 shows the thermal expansion coefficients of materials used for sensor die and sensor housing.

Table 2 Thermal expansion coefficients of materials used for pressure sensors

\begin{tabular}{|l|l|l|}
\hline Field of application & Material & Thermal expansion coefficient \\
\hline sensor element & silicon & $3,610^{-6} / \mathrm{K}$ \\
\hline & glass & $2,810^{-6} / \mathrm{K}$ \\
\hline substrate & kovar $\mathrm{AL}_{2} \mathrm{O}_{3}$ & $510^{-6} / \mathrm{K}$ \\
\hline & ceramic & $6,3910^{-6} / \mathrm{K}$ \\
\hline & ceramic $\mathrm{HTCC}$ & $5,810^{-6} / \mathrm{K}$ \\
\hline adhesives & aluminium & $2410^{-6} / \mathrm{K}$ \\
\hline & epoxy adhesives & $2310^{-6} / \mathrm{K}$ \\
\hline
\end{tabular}

At the cure temperature of the adhesive the sensor - substrate sandwich is stress free. Due to the mismatch of thermal expansion of sensor, substrate and adhesive the cooling after cure leads to stress. The adhesive bonds for the mounting processes can be classified in two groups: soft silicone, fluorine silicone bonds and hard epoxy bonds. Table 3 shows the properties of typical adhesive bonds.

Table 3 Typical properties of adhesive bonds

\begin{tabular}{|l|l|l|l|l|}
\hline Adhesive bond & Silicone & $\begin{array}{l}\text { Fluorine } \\
\text { Silicone }\end{array}$ & Epoxy & $\begin{array}{l}\text { Quarz Filled } \\
\text { Epoxy }\end{array}$ \\
\hline E-Module & - & $5,9 \mathrm{MPa}$ & $3200 \mathrm{MPa}$ & $12000 \mathrm{MPa}$ \\
\hline Durometer Hardness Shore A & 30 & 35 & - & - \\
\hline Elongation & $550 \%$ & $425 \%$ & $1,2 \%$ & $0,3 \%$ \\
\hline Tensile Strength & $4,6 \mathrm{MPa}$ & $2,6 \mathrm{MPa}$ & $50 \mathrm{MPa}$ & $40 \mathrm{MPa}$ \\
\hline Temperature range & & -65 to $240{ }^{\circ} \mathrm{C}$ & -40 to $150{ }^{\circ} \mathrm{C}$ & -40 to $150{ }^{\circ} \mathrm{C}$ \\
\hline Glasstemperature & - & - & $146{ }^{\circ} \mathrm{C}$ & $175^{\circ} \mathrm{C}$ \\
\hline
\end{tabular}

The soft adhesive bonds have the advantage that they are able to decouple the thermal mismatch of substrate and sensor die. When they have thixotropic properties they are deposited in a thick layer, equalising the different elongations. Because of the low hardness only low mechanical stress is implied in the sensor die. Disadvantage of this mounting is the reduced strength of the junction. A hard adhesive bond has to be used for applications with pressure over 10 bar. Because the adhesive is not able to compensate the thermal mismatch between glass and the substrate, a large mechanical stress is implied. The tensil forces can destroy the glass of the sensor die occurring during the holohedral bonding of glass and aluminum with epoxy. At small defects caused by the wafer dicing process fissures starts and crush the glass.

To reduce the implied mechanical tensions hard bonded sensor elements are mounted on shafts, made by materials with an adapted thermal coefficient like kovar or ceramic.

One detrimental property of the adhesives is their affinity for diffusions reactions catching ions of pressure leading media. Silicone adhesives, in particular, can enlarge their volume. The swelling starts at the side 
with media contact and implies forces in the sensor element. This tensions result in a drift of the offset voltage. Fig. 2 shows the offset drift while the swelling process of different adhesive bonds.

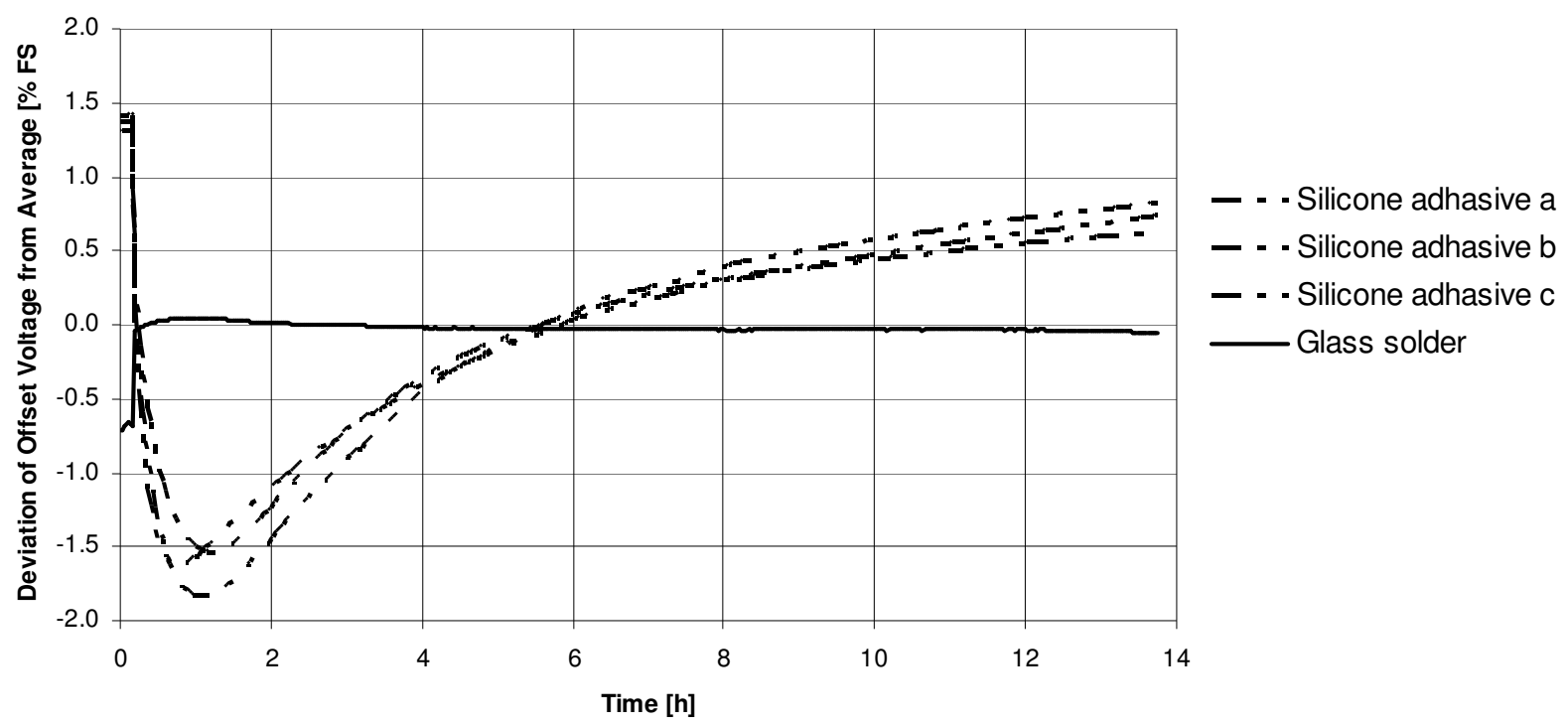

Fig. 2: Swelling caused offset drift of different adhesive bondings

Hysteresis and drift errors that cannot be compensated are also caused by the glass temperature of adhesives. Silicone and fluoridic silicone adhesives have a low glass temperature, so they are used in the range above this temperature. At low temperatures the adhesives solidify and cause increasing mechanical tensions with hysteresis effects.

Hard epoxy adhesives are applicated in the range below their glass temperature. Above the glass temperature the adhesives get softer and have an enlarged thermal expansion. The mechanical tensions can release and are fixed by cooling down at another state. So the offset voltage of the Wheatstone bridge shows a drift and hysteresis behavior Figure 3 shows the offset behavior of different mountings of sensor elements on a ceramic substrate.

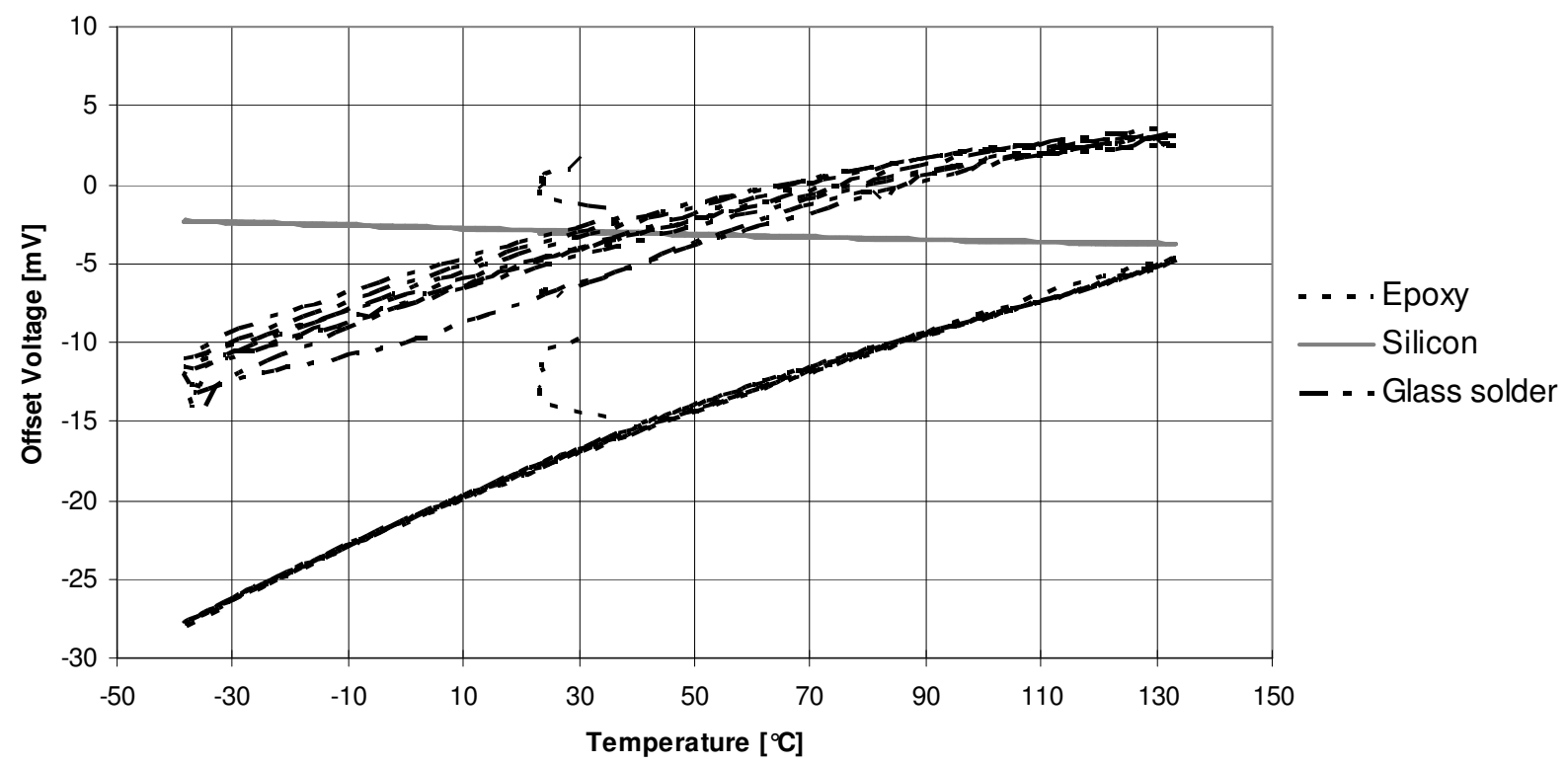

Fig. 3 Offset signal of sensor dies mounted on ceramic substrates in a temperature range from $-40{ }^{\circ} \mathrm{C}$ to 135 ${ }^{\circ} \mathrm{C}$, while 5 cycles.

The problems of media resistance and undefinite mechanical behavior of adhesives can be avoided by mounting the pressure sensor die with glass soldering. If the sensor element is discharged with pressure 
from the massive silicon backside the sensor is resistant against fuel, alcohol and other petrochemical solvents can be verified. The glass soldering process brings mechanical tensions in, but they are frozen in the system by the high glass temperature. Fig. 3 shows the offset behavior of different mountings of sensor elements on a ceramic substrate.

\section{Electrical interconnect of the sensor element}

The bondpads for the electrical connection of the sensor to the system are on the topside of the chip, where the high doped circuits and the piezoresistive Wheatstone bridge are placed. Because of the proximity of the sensing elements the input of mechanical tensions must be avoided. Since the first piezoresistive pressure elements wire bonding is state of the art for pressure die interconnect. In stead of flip chip mounting wire bonds don't cause mechanical tensions.

New solutions use structures of movable fingers for stressless interconnect of sensor dies. For example a flexible metallised kapton film for contacting and mounting the absolute pressure sensor is used. This technology was developed as TAB-technology (TAB: Tape Automated Bonding) by Fa. Keller [5]. New developments and alternatives to the wire bond technology will contribute to miniaturization of pressure transmitter systems.

\section{Encapsulation of the sensor element}

The topside of the silicon sensor element is highly sensitive to corrosion and diffusion effects. Aluminum bond pads and wire bonds combined with the signals voltage allow electrochemical corrosion. Ions can destroy the covering oxide layers and diffuse in the silicon with its doped resistors. To protect this corrosion sensitive area against contamination it is covered with a silicone gel or encapsulated in an oil filled sensor cell covered with a diaphragm of high alloyed steel. In most applications of pressure transmitters air or minor contaminated air is in contact to the sensor element so the gel covering provides a sufficient protection. Media-separated solutions have advantages in applications with aggressive media and high demands on the long-term stability, but they also involve also a higher technical effort and expense [3].

The silicon sensor die and the silicone gel show different thermal behavior. This difference is compensated by the rheologic properties of the gel, flowing in a stressless geometry. Condition for that behavior is that the gel has enough volume fore expansion. In miniaturized housings the volume is reduced and the sensor element is enclosed by the housings walls. The influence of the aspect of the chamber between the sensor element and wall are investigated in an experiment. A sensitive pressure sensor element of $3 \times 3 \mathrm{~mm}^{2}$ is mounted with a distance of $0.5,1$ and $1,5 \mathrm{~mm}$ from the outer walls of a ceramic housing and covered with an silicone gel. The offset voltage is measured 10 times in a -40 to 135 ${ }^{\circ} \mathrm{C}$ cycle. The results are presented in Fig. 4.

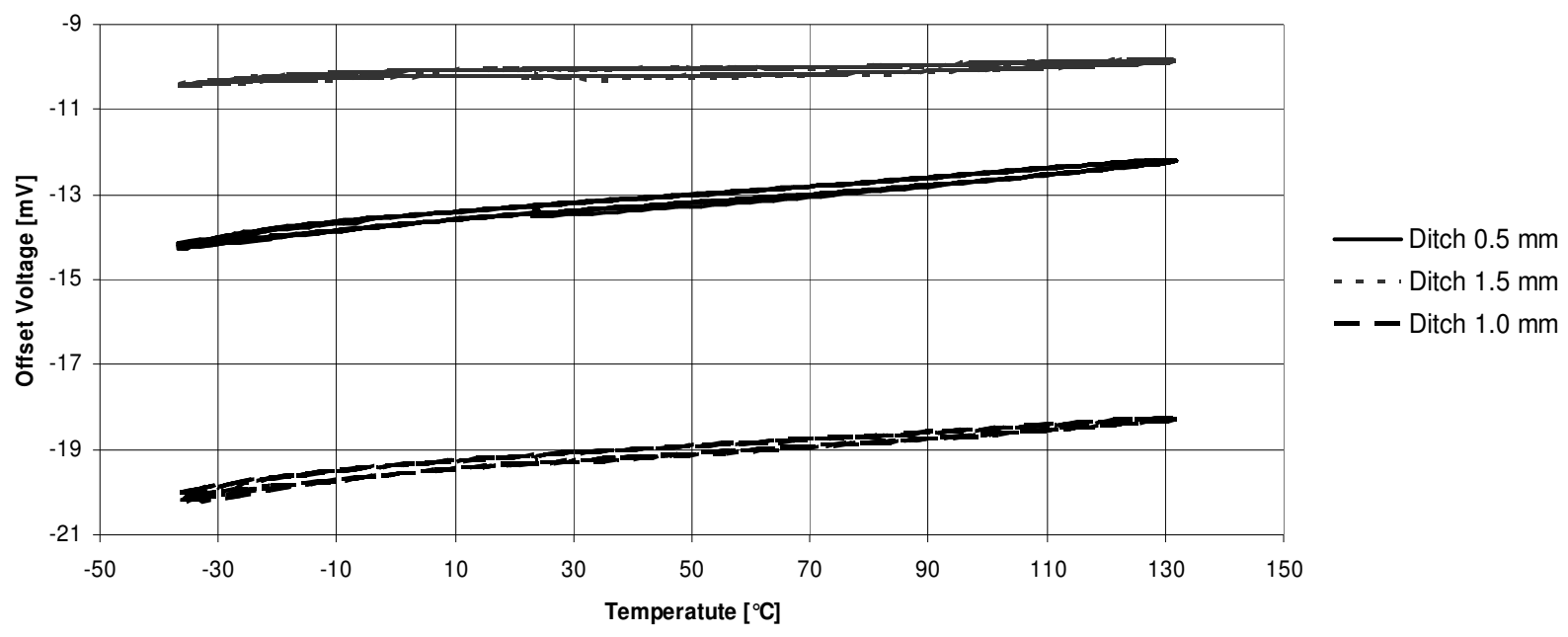

Fig. 4 Offset behavior of piezoresistive pressure sensors with different ditches 


\section{Construction of the miniaturized pressure transmitter}

The mounting and coating of piezoresistive pressure sensor elements were presented. For a successful realization of an integrated pressure sensor system additional requirements must be considered. The demands to the sensor housing are:

- mechanical rugged structure in spite of inlets and channels

- $\quad$ well controlled thermo-mechanical properties with small temperature hysteresis

- $\quad$ decoupling of the sensor die from internal and external stress

- $\quad$ the ability for integration of electrical functions

- $\quad$ process suitable for mass production (cost reduction)

These features are offered by high temperature co-fired ceramics (HTCC). Considering the effects of mounting to the sensor die a HTCC package for relative and differential pressure was developed. In the production process each layer of the ceramic is punched, connections and vias are formed with conductive paste by printing, all layers are assembled and fired to form a compact structure. Finally, the housings are separated by dicing. The package for the relative and differential pressure sensor has outer dimensions of $13 \times 16 \mathrm{~mm}^{2}$. Two cavities are integrated in the ceramic, one for the sensor element and one for the signal conditioner. Fig. 5 shows different views of the differential pressure transmitter.

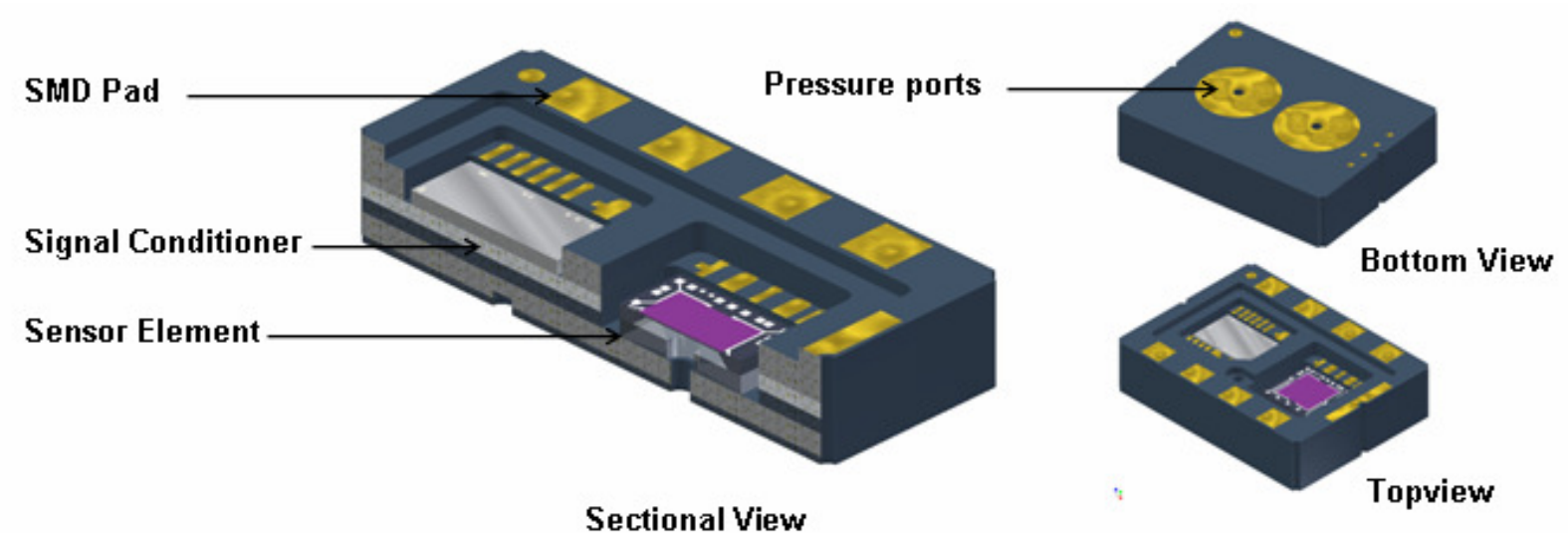

Fig. 5 Different views of the miniaturizminiaturized differential pressure sensor package

The piezoresistive sensor element features a glass plate on its bottom side for reducing mounting stress. It is bonded with soft silicone adhesive in the larger cavity. Between the sensor element and the walls a distance is kept to enable thermal expansion of the coating gel for preventing environmental stress. One pressure is applied at the topside of the sensor element, the second pressure is lead thru a channel to the backside. The pressure ports can be adapted to customer specifications and are placed on the topside. The signal conditioner is mounted as bare die in the second cavity. Both silicon elements are contacted with wire bonds.

The cavities are closed by an adhesive bonded ceramic plate. On the bottom side of the ceramic housing solder bumps are provided for direct SMD-mounting.

The nominal pressure is between $100 \mathrm{mbar}$ and 10 bar. Different piezoresistive sensor elements can be applied. Signal conditioners are available for analog ( 0.5 to $4.5 \mathrm{~V})$ or digital ( ${ }^{2} \mathrm{C}$ bus) output.

\section{Barometric pressure sensor}

At EPCOS advanced packaging technologies for SAW-Filters (Surface Acoustic Wave) were developed in the last two decades [3]. Like SAW-Filters pressure sensors and other MEMS components need packages with cavities above the chip surface so our SAW packaging know-how was applied to MEMS components. This new MEMS packaging technology was named CSMPTM (chip-sized MEMS package) [6].

For miniaturisation a piezoresistive pressure sensor chip with a size of only $1 \times 1 \mathrm{~mm}^{2}$ was developed. The worlds smallest fully packaged barometric pressure sensor with dimensions of only $1.7 \times 1.7 \times 0.9$ $\mathrm{mm}^{3}$ was made by packaging this sensor die with CSMPTM technology. The primary application is the measuring of air pressure in electronic equipment for example in portable navigation devices, hard disks, 
watches and mobile phones. The resolution and the noise ratio of the pressure sensor allow to measure air pressure and/or altitude above sea level.

For other applications EPCOS also offers a sensor in a standard package with gel protection and a stainless steel pressure port. With a footprint of only $3 \times 3 \mathrm{~mm}^{2}$ this pressure sensor enables the further miniaturization of barometric applications that are exposed to high humidity or other modest contaminations. Fig. 6 shows the miniaturised barometric pressure sensors T5000 and ASB 1200E.

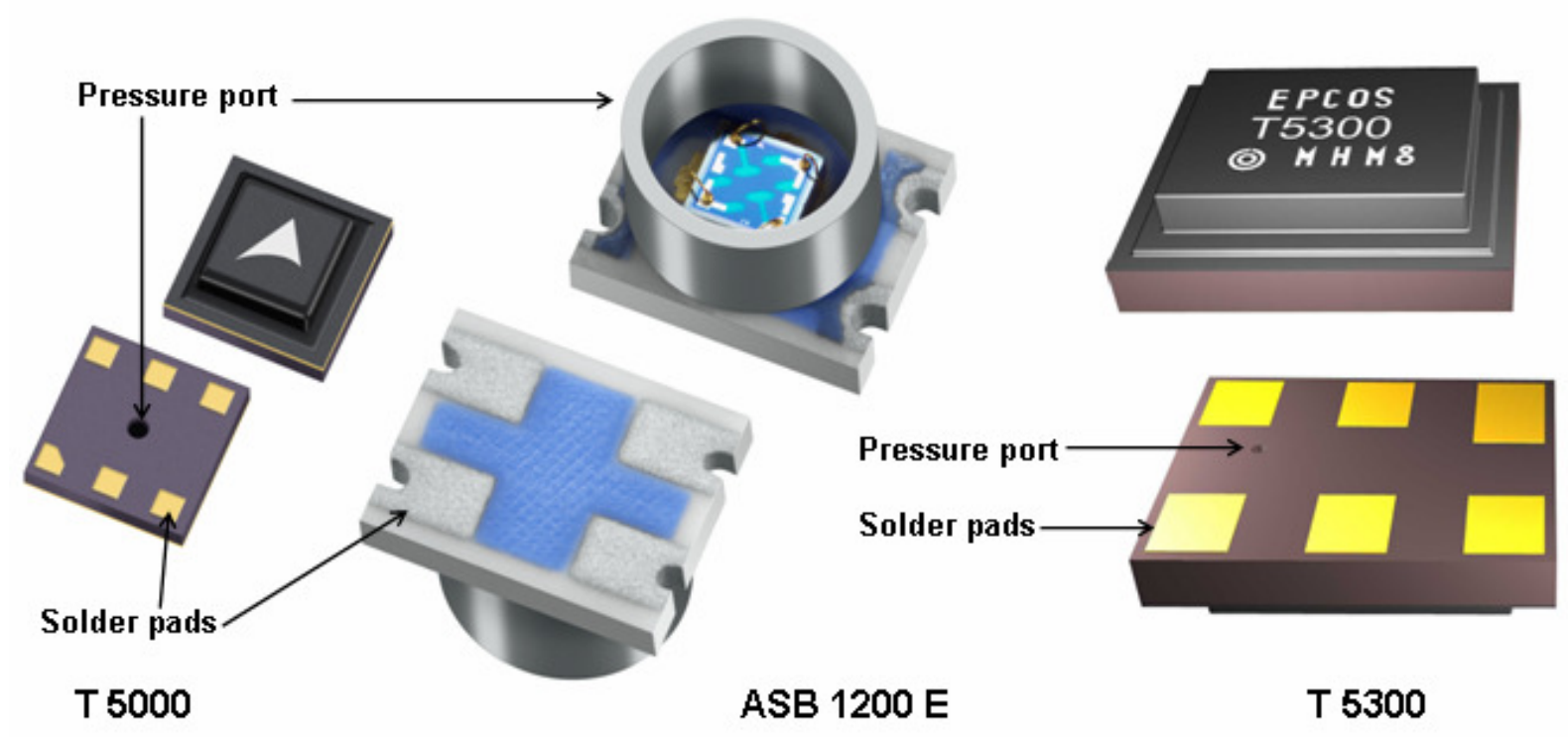

Fig. 6 T5000, ASB1200E barometric pressure sensors and T 5300 pressure transmitter

Both pressure sensors are optimized for an absolute pressure measurement from 300 to $1200 \mathrm{mbar}$ and show a transmission ratio of $20 \mathrm{mV} / \mathrm{V} / \mathrm{bar}$.

T5000 and ASB 1200E are packaged sensor dies. For most consumer applications a calibrated sensor system with digital interface is required. So a CSMPTM pressure sensor with integrated signal conditioner was developed. The package size is $2.2 \times 2.6 \mathrm{~mm}^{2}$ with a height of only $0.8 \mathrm{~mm}$. So this highly miniaturised sensor system is well suited for portable devices like navigation systems or mobile phones. The sensor die is mounted with a technology for low stress flip chip assembly so hysteresis and drift are minimised.

\section{References}

[1] Gerlach, G., Werthschützky, R.: 50 Jahre Entdeckung des piezoresistiven Effekts - Geschichte und Entwicklungsstand piezorsistiver Sensoren. tm - Technisches Messen 02.05, Oldenbourg Wissenschaftsverlag 2005

[2] Gevatter, H.,Automatisierungstechnik 1 - Mess- und Sensortechnik. Springer Verlag, Berlin 2000

[3] Kaden, G, Das Packaging, der Schlüssel für die Nutung des piezoresitiven Drucksensors. tm Technisches Messen 72 (2005) 12/2005

[4] Deier, E.; Hoyden, J.;Wilde, J.; Becker, K. : Thermodynamische Einflüsse der Chipklebung auf die Genauigkeit mikromechanischer Drucksensoren. tm - Technisches Messen 72 (2005) 2/2005

[5] Kanz, J; Braun, G.; Unger, R.: Bumped Tape Automated Bonding (BTAB). Components, Hybrids and manufacturing Technology, IEEE Transactions on Vol.2, Issue 3, Sep 1979 Page 301 - 308

[6] Feiertag, G.; Krüger, H.; Bauer, C.: Surface Acoustic Wave Component Packaging. Proceedings 16th European Microelectronics and Packaging Conference (EMPC), Oulu, Finnland, July 2007 